\title{
Comprehensive Solution for Transient Flow in Heterogeneous Porous Media
}

\author{
Ricardo Hüntemann Deucher ${ }^{1}$. Paulo Couto ${ }^{2}$. \\ Gustavo César Rachid Bodstein ${ }^{3}$
}

Received: 4 December 2015 / Accepted: 13 May 2016 / Published online: 28 May 2016

(C) Springer Science+Business Media Dordrecht 2016

\begin{abstract}
Solutions of the hydraulic diffusivity equation are of utmost importance for many reservoir engineering problems. Despite all the efforts, there is still a need for the development of rigorous and comprehensive solutions for transient flow problems in heterogeneous oil reservoirs. This study demonstrates the use of an integral transform approach to obtain such a rigorous and comprehensive solution for the hydraulic diffusivity equation in heterogeneous porous domain. The reservoir heterogeneities can be approximated by any continuous differentiable function. The presented general solution and its derivation are valid for multidimensional problems in any orthogonal coordinate system. It has the advantage of rigorously solving the hydraulic diffusivity equation for transient, late-transient and steady-state (or pseudo-steady-state) flow regimes in a single formulation that allows the consideration of variable flowrates. In this work, applications of the general solution for one-dimensional problems in the Cartesian and radial coordinate systems are presented, showing comparisons of the results obtained with a finite difference numerical scheme. The solution presented can be used to analyze buildup, drawdown and interference test data, making it a useful tool for pressure transient analysis applied to reservoir engineering problems.
\end{abstract}

Keywords Analytical solution · Hydraulic diffusivity · Integral transform technique · Transient flow $\cdot$ Porous media

$\triangle$ Ricardo Hüntemann Deucher rhdeucher@gmail.com

1 Petrobras S/A, Av. Republica do Chile 330, Centro, Rio de Janeiro, Brazil

2 Civil Engineering Program, COPPE, Federal University of Rio de Janeiro (UFRJ), Rio de Janeiro, Brazil

3 Mechanical Engineering Program, COPPE, Federal University of Rio de Janeiro (UFRJ), Rio de Janeiro, Brazil 


\section{List of symbols}

$\begin{array}{ll}L_{x}, L_{y}, L_{z}, r_{e} & \text { Reservoir dimensions } \\ N_{\mathrm{i}} & \text { Normalization integrals } \\ c_{\mathrm{t}} & \text { Total compressibility } \\ p_{\mathrm{i}} & \text { Initial pressure } \\ F(\boldsymbol{x}) & \text { Initial condition } \\ a(\boldsymbol{x}), b(\boldsymbol{x}) & \text { Boundary condition coefficients } \\ k & \text { Permeability } \\ p & \text { Pressure } \\ q & \text { Flowrate } \\ x_{\mathrm{p}}, r_{\mathrm{p}} & \text { Wellbore position } \\ W_{\mathrm{p}} & \text { Source term } \\ t & \text { Time } \\ \boldsymbol{x} & \text { Position vector } \\ S & \text { Domain surface } \\ V & \text { Domain region } \\ x_{c} & \text { Parameter of Equation (14) } \\ r_{p} & \text { Oil well radius }\end{array}$

\section{Greek symbols}

$\begin{array}{ll}\eta & \text { Hydraulic diffusivity coefficient } \\ \mu & \text { Viscosity } \\ \phi & \text { Porosity } \\ \phi(x, t) & \text { Boundary condition coefficients } \\ \psi(\boldsymbol{x}) & \text { Heterogeneous problem eigenfunctions } \\ \sigma & \text { Heterogeneous problem eigenvalues } \\ \Omega(\boldsymbol{x}) & \text { Auxiliary problem eigenfunctions } \\ \lambda & \text { Auxiliary problem eigenvalues } \\ \text { erf } & \text { Error function } \\ \text { erfc } & \text { Complementary error function } \\ E_{\mathrm{i}} & \text { Exponential integral function } \\ \delta & \text { Dirac delta function }\end{array}$

\section{Introduction}

Solutions of the hydraulic diffusivity equation (HDE) have been a subject of study of the petroleum industry since the first half of the twentieth century. The development of solutions for the HDE is of utmost importance for many reservoir engineering problems, since the HDE describes the transient pressure behavior in the porous media. A commonly employed form of the HDE is given by:

$$
\frac{1}{\eta} \frac{\partial p(\boldsymbol{x}, t)}{\partial t}=\nabla^{2} p(\boldsymbol{x}, t)
$$


where $\eta$ is the hydraulic diffusivity coefficient given by $\eta=k / \phi \mu c_{\mathrm{t}}$. Solutions of Eq. 1 form the foundation of pressure analysis techniques applied to reservoir engineering problems (Matthews and Russell 1967).

The formulation given by Eq. 1 considers a set of simplifying assumptions: (1) fluid of small and constant compressibility (most oilfield liquids fit this assumption properly); (2) constant fluid viscosity; (3) homogeneous and isotropic porous media; (4) Darcy's law is respected; (5) small pressure gradients; and (6) negligible gravity forces. In the formulation given by Eq. 1, the well effects are usually taken into account in the boundary conditions.

According to Cotta (1993), analytical solutions present advantages when compared to numerical solutions: They are more exact and generally much less computationally expensive, provide trends and asymptotic behaviors, allowing for straightforward inspections of parameter variations, and can provide benchmark results for the validation of numerical schemes. Carslaw and Jaeger (1959) presented analytical solutions of Eq. 1 that are widely employed in the reservoir engineering literature for both the Cartesian and radial coordinate systems, respectively:

$$
\begin{aligned}
& p(x, t)=p_{\mathrm{i}}-\frac{q \mu}{k L_{y} L_{z}}\left[x \operatorname{erfc}\left(\frac{x}{\sqrt{4 \eta t}}\right)-\sqrt{\frac{4 \eta t}{\pi}} \mathrm{e}^{-\frac{x^{2}}{4 \eta t}}\right] \\
& p(r, t)=p_{\mathrm{i}}-\frac{q \mu}{2 \pi k L_{z}}\left[\frac{1}{2} \operatorname{Ei}\left(-\frac{r^{2}}{4 \eta t}\right)\right]
\end{aligned}
$$

Despite their importance for many reservoir engineering problems, the solutions given by Eqs. 2-3 are limited to very restricted cases, as these solutions consider: (1) one-dimensional fluid flow in a semi-infinite porous media; (2) constant flowrate; (3) fully penetrating wells; and (4) uniform initial condition.

The classical integral transform technique (CITT) is a method for the solution of linear diffusion problems based on the method of separation of variables, which expands the solution in terms of expansions of an auxiliary eigenvalue problem. The use of the CITT to obtain analytical solutions of seven classes of linear diffusion problems has been presented and analyzed by Mikhailov and Ozisik (1984). The obtained formal solutions are applicable over a broad range of problems.

Aiming to obtain more general solutions of the HDE, Hovanessian (1961) presented the use of the CITT to analytically solve the transient HDE considering the flow of slightly compressible liquids through a two-dimensional homogeneous porous media in the Cartesian coordinate system. Numerical examples were given to illustrate the application of the solution to practical problems.

Rahman and Bentsen (2000) presented a systematic approach to obtain several solutions of the HDE by CITT in the Cartesian coordinate system for one-, two- and three-dimensional problems, with all possible combinations of the boundary conditions. Later, Rahman and Bentsen (2001) extended the systematic approach to obtain analytical solution of the HDE by CITT to problems in the radial coordinate system. In these two works, the application of the technique was not demonstrated.

Despite all the advances attained by Hovanessian (1961), Rahman and Bentsen (2000), Rahman and Bentsen (2001) and many others, there is still a need for the development of rigorous and comprehensive solutions for transient flow problems in heterogeneous oil reservoirs.

An extension of the analytical ideas behind the CITT led to the development of the generalized integral transform technique (GITT) (Cotta 1993), which offers more flexibility in handling a priori non-transformable problems, allowing the solution of more involved 
situations, as the solution of nonlinear (Cotta 1994) convection-diffusion problems (Almeida and Cotta 1995). Naveira-Cotta et al. (2009) presented the use of the GITT to obtain analytical solutions of eigenvalue problems related to diffusion in heterogeneous media. Following these ideas, Couto et al. (2011) presented a formal and generalized solution of the HDE in heterogeneous porous media, without presenting applications of the proposed solution to heterogeneous domains.

This work presents the use of the GITT to attain rigorous and comprehensive solutions of transient flow problems in heterogeneous reservoirs. Applications of the proposed solution to transient flow problems in the Cartesian and radial coordinate systems will illustrate the accuracy of the proposed solution.

\section{Problem Formulation}

A general three-dimensional formulation of the HDE that considers transient fluid flow through heterogeneous reservoirs will be employed in this work. This formulation can be given by:

$$
\phi(\boldsymbol{x}) c_{\mathrm{t}} \frac{\partial p(\boldsymbol{x}, t)}{\partial t}=\nabla \cdot\left(\frac{k(\boldsymbol{x})}{\mu} \nabla p(\boldsymbol{x}, t)\right)+W_{\mathrm{p}}(\boldsymbol{x}, t), \quad \boldsymbol{x} \in V, t>0
$$

with generalized boundary conditions:

$$
a(\boldsymbol{x}) p(\boldsymbol{x}, t)+b(\boldsymbol{x}) \frac{k(\boldsymbol{x})}{\mu} \frac{\partial p(\boldsymbol{x}, t)}{\partial \boldsymbol{n}}=\varphi(\boldsymbol{x}, t), \quad \boldsymbol{x} \in S, t>0
$$

and generalized initial condition:

$$
p(\boldsymbol{x}, t)=F(\boldsymbol{x}), \quad \boldsymbol{x} \in V, t=0
$$

The parameters $a(\boldsymbol{x})$ and $b(\boldsymbol{x})$ can be handled in order to obtain boundary conditions of the 1st kind (prescribed pressure, $b(\boldsymbol{x})=0$ ), 2nd kind (prescribed flux or no flow boundary, $a(\boldsymbol{x})=0$ ) and of the 3rd kind. Most of the reservoir engineering applications involve boundary conditions of the 1st and 2nd kind, which are also called Dirichlet and Newman boundary conditions, respectively.

The initial condition given by Eq. (4c) is general and can be chosen in order to represent an initially uniform pressure distribution or any other initial pressure distribution considered adequate to the flow problem that is going to be solved.

In the way it is written, Problem (4) neglects gravitational effects. This simplification can be removed by considering that $p(\boldsymbol{x}, t)$ represents flow potential instead of pressure. In Problem (4), $\boldsymbol{x}$ is the position vector according to any orthogonal coordinate system, $k(\boldsymbol{x})$ is the permeability coefficient, $\phi(\boldsymbol{x})$ is the porosity coefficient, and $W_{\mathrm{p}}(\boldsymbol{x}, t)$ is a source term that represents the well production. $W_{\mathrm{p}}(\boldsymbol{x}, t)$ has dimensions of $s^{-1}$ and is given by:

$$
W_{\mathrm{p}}(\boldsymbol{x}, t)=\frac{q(t)}{A} \delta\left(\boldsymbol{x}-\boldsymbol{x}_{\mathrm{p}}\right)
$$

where $q(t)$ is the volumetric flowrate in reservoir conditions that can be represented by any continuous differentiable function, $\delta$ is the Dirac delta function, and $\boldsymbol{x}_{\mathrm{p}}$ represents the well position inside the reservoir. The parameter $A$ depends on the coordinate system employed as well as on the number of dimensions that are going to be considered. The definition of the parameter $A$ is straightforward and can be found in Rahman and Bentsen (2000) for the 
Cartesian coordinate system and in Rahman and Bentsen (2001) for the radial coordinate system.

The rigorous solution of Eq. 4a allows one to use the space- dependent coefficients $\phi(\boldsymbol{x})$ and $k(\boldsymbol{x})$ to represent heterogeneities of the porous medium, as layered formations, sealing or conducting faults, skin or damage effects, etc. The space- and time-dependent source term can be used to represent production of a well with variable flowrate as well as the production of more than one well inside the same reservoir (only for Cartesian coordinate system).

\section{Solution Methodology}

The solution of the generalized problem given by Eqs. $4 \mathrm{a}-4 \mathrm{c}$ by the GITT considers two steps: (1) solution of Problem (4) itself using the CITT and (2) solution of the associated eigenvalue problem with variable coefficients.

\section{Step 1: Solution of Problem (4) using the CITT}

Following the formalisms presented by Mikhailov and Ozisik (1984), an exact solution of Problem (4) by the classical integral transform technique can be obtained:

$$
p(\boldsymbol{x}, t)=\sum_{i=1}^{\infty} \hat{\psi}_{i}(\boldsymbol{x})\left(\bar{f}_{i} \mathrm{e}^{-\sigma_{i}^{2} t}+\int_{0}^{t} \bar{g}_{i}\left(t^{\prime}\right) \mathrm{e}^{-\sigma_{i}^{2}\left(t-t^{\prime}\right)} \mathrm{d} t^{\prime}\right)
$$

The eigenvalues and eigenfunctions are obtained from the eigenvalue problem that contains the information regarding the reservoir heterogeneity. This eigenvalue problem is obtained by the separation of variables of the homogeneous version of Problem (4) and can be written as:

$$
\nabla \cdot\left(\frac{k(\boldsymbol{x})}{\mu} \nabla \psi_{i(\boldsymbol{x})}\right)+\phi(\boldsymbol{x}) c_{\mathrm{t}} \sigma_{i}^{2} \psi_{i(\boldsymbol{x})}=0, \quad \boldsymbol{x} \in V
$$

with homogeneous boundary conditions given by:

$$
a(\boldsymbol{x}) \psi_{i(\boldsymbol{x})}+b(\boldsymbol{x}) \frac{k(\boldsymbol{x})}{\mu} \frac{\partial \psi_{i(\boldsymbol{x})}}{\partial \boldsymbol{n}}=0, \quad \boldsymbol{x} \in S
$$

The normalized eigenfunctions as well as the transformed source terms and initial condition are computed with Eqs. 8a-8d after solving the eigenvalue problem given by Eqs. 7a-7b.

$$
\begin{aligned}
& \hat{\psi}_{i}(\boldsymbol{x})=\frac{\psi_{i(\boldsymbol{x})}}{N_{\mathrm{i}}^{1 / 2}} \quad \text { Normalized eigenfunctions } \\
& N_{\mathrm{i}}=\int_{V} \phi(\boldsymbol{x}) c_{\mathrm{t}} \psi_{\mathrm{i}}^{2}(\boldsymbol{x}) \mathrm{d} V \quad \text { Normalization integrals } \\
& \bar{f}_{i}=\int_{V} \phi(\boldsymbol{x}) c_{\mathrm{t}} F(\boldsymbol{x}) \hat{\psi}_{i}(\boldsymbol{x}) \mathrm{d} V \quad \text { Transformed initial condition } \\
& \bar{g}_{i}(t)=\int_{V} W_{\mathrm{p}}(\boldsymbol{x}, t) \hat{\psi}_{i}(\boldsymbol{x}) \mathrm{d} V+\int_{S} \varphi(\boldsymbol{x}, t)\left[\frac{\hat{\psi}_{i}(\boldsymbol{x})-\frac{k(\boldsymbol{x})}{\mu} \frac{\partial \hat{\psi}_{i}(\boldsymbol{x})}{\partial \boldsymbol{n}}}{a(\boldsymbol{x})+b(\boldsymbol{x})}\right] \mathrm{d} S
\end{aligned}
$$

Transformed source term

The source term $\bar{g}_{i}(t)$ includes the information of both the well's production and the boundary conditions. It is important to note that the existence of source terms in the bound- 
aries of the reservoir (non-homogeneous boundary conditions) causes difficulties to obtain a converged solution at these boundaries, making it important to use filter solutions in order to improve the convergence behavior.

\section{Step 2: Solution of the associated eigenvalue problem with variable coefficients}

The eigenvalue problem containing the information related to the heterogeneous media, Problem (7), will be tackled with the generalized integral transform technique by the proposition of an appropriate basis that consists of a simpler auxiliary eigenvalue problem with readily available solutions, and expanding the solution of Problem (7) in terms of this simpler auxiliary eigenvalue problem is given by:

$$
\begin{aligned}
& \nabla \cdot\left(\frac{k^{*}(\boldsymbol{x})}{\mu} \nabla \Omega_{n(\boldsymbol{x})}\right)+\phi^{*}(\boldsymbol{x}) c_{\mathrm{t}} \lambda_{n}^{2} \Omega_{n(\boldsymbol{x})}=0, \boldsymbol{x} \in V \\
& a(\boldsymbol{x}) \Omega_{n(\boldsymbol{x})}+b(\boldsymbol{x}) \frac{k^{*}(x)}{\mu} \frac{\partial \Omega_{n(\boldsymbol{x})}}{\partial \boldsymbol{n}}=0, \quad \boldsymbol{x} \in S
\end{aligned}
$$

where the coefficients $k^{*}(\boldsymbol{x})$ and $\phi^{*}(\boldsymbol{x})$ are simpler forms of the function that represents the heterogeneous distribution of permeability and porosity throughout the reservoir. The forms of $k^{*}(\boldsymbol{x})$ and $\phi^{*}(\boldsymbol{x})$ are chosen in order to allow for an analytical solution of Problem (9). A simple choice of these coefficients is $k^{*}(\boldsymbol{x})=k_{\mathrm{avg}}$ and $\phi^{*}(\boldsymbol{x})=\phi_{\mathrm{avg}}$, where $k_{\mathrm{avg}}$ and $\phi_{\mathrm{avg}}$ represent the average reservoir permeability and porosity, respectively. For this situation, the eigenfunctions $\Omega_{n(x)}$ may be obtained by separation of variables for a general multidimensional formulation and the solution of the separated problems can be readily obtained from tabulated solutions of classical eigenvalue problems (Ozisik 1993).

The solution of Problem (9) is used as a basis for the eigenfunction expansion of the eigenvalue Problem (7), which contains the information regarding the reservoir heterogeneity:

$$
\begin{aligned}
\psi_{i(\boldsymbol{x})} & =\sum_{n=1}^{\infty} \hat{\Omega}_{n(\boldsymbol{x})} \hat{\psi}_{i, n} \quad \text { Inverse } \\
\hat{\psi}_{i, n} & =\int_{V} \phi^{*}(\boldsymbol{x}) c_{\mathrm{t}} \psi_{i(\boldsymbol{x})} \hat{\Omega}_{n(\boldsymbol{x})} \mathrm{d} V \quad \text { Transform }
\end{aligned}
$$

The integral transformation of Problem (7) is performed by following the formalisms of the GITT, according to Cotta (1993), in order to obtain:

$$
\begin{aligned}
& \sum_{m=1}^{\infty} \hat{\psi}_{i, m}\left[\int_{V} \hat{\Omega}_{m(\boldsymbol{x})}\left(\nabla \cdot \frac{k(\boldsymbol{x})}{\mu} \nabla \hat{\Omega}_{n(\boldsymbol{x})}\right) \mathrm{d} V+\int_{S} \frac{k(\boldsymbol{x})}{\mu}\left(1-\frac{k^{*}(\boldsymbol{x})}{k(\boldsymbol{x})}\right)\left(\hat{\Omega}_{m(\boldsymbol{x})} \frac{\partial \hat{\Omega}_{n(\boldsymbol{x})}}{\partial \boldsymbol{n}}\right) \mathrm{d} s\right. \\
& \left.+\int_{V} \hat{\Omega}_{m(\boldsymbol{x})} \sigma_{i}^{2} \frac{k^{*}(\boldsymbol{x})}{\mu} \hat{\Omega}_{n(\boldsymbol{x})} \mathrm{d} V\right]=0
\end{aligned}
$$

The eigensystem given by Eq. 11 can be concisely written in matrix form:

$$
\begin{aligned}
& \left(\boldsymbol{A}-\sigma^{2} \boldsymbol{B}\right) \hat{\boldsymbol{\psi}}=0 ; \hat{\boldsymbol{\psi}}=\left\{\hat{\psi}_{n, m}\right\} ; \boldsymbol{B}=\left\{B_{n, m}\right\} ; \boldsymbol{A}=\left\{A_{n, m}\right\} \\
& B_{n, m}=\int_{V} \hat{\Omega}_{m(\boldsymbol{x})} \sigma_{i}^{2} K_{(\boldsymbol{x})} \frac{k^{*}(\boldsymbol{x})}{\mu} \hat{\Omega}_{n(\boldsymbol{x})} \mathrm{d} V \\
& A_{n, m}=\int_{V} \hat{\Omega}_{m(\boldsymbol{x})}\left(\nabla \cdot \frac{k(\boldsymbol{x})}{\mu} \nabla \hat{\Omega}_{n(\boldsymbol{x})}\right) \mathrm{d} V+\int_{S} \frac{k(\boldsymbol{x})}{\mu}\left(1-\frac{k^{*}(\boldsymbol{x})}{k(\boldsymbol{x})}\right)\left(\hat{\Omega}_{m(\boldsymbol{x})} \frac{\partial \hat{\Omega}_{n(\boldsymbol{x})}}{\partial \boldsymbol{n}}\right) \mathrm{d} s
\end{aligned}
$$


The algebraic Problem (12) is solved to provide results for the eigenvalues $\left(\sigma^{2}\right)$ and eigenfunctions $(\hat{\psi})$, which can be combined with the inverse formula (10a) in order to provide the eigenfunctions that contain the information associated with reservoir heterogeneity. After one obtains the solution of the eigenvalue problem, it is possible to use the solution given by Eq. 6 in order to obtain the transient pressure behavior in a heterogeneous reservoir.

For a homogeneous reservoir, the solution given by Eq. 6 is still valid, and instead of using the procedure presented in Step 2 to solve the eigenvalue problem with variable coefficients, one can directly call for tabulated solutions of the associated eigenvalue problem with constant coefficients, as the ones presented in the textbook by Ozisik (1993).

If analytical representation of the integrals involved in Eqs. (6), (8a), (11) and (12a) are not available, it will be necessary to numerically integrate these terms. This task, which was only necessary for the radial coordinate system, can be performed by consolidated routines with controlled accuracy, such as the Gaussian quadrature.

\section{Applications}

Applications of the proposed approach to solve the HDE for one-dimensional problems in the Cartesian and radial coordinate systems will be presented in this section. The solution was implemented in the platform Mathematica 9.0 (Wolfram 2005). Although not presented in this work, the computational implementation can be extended in future works to two- or three-dimensional problems, given an adequate reordering of the terms in the eigenfunction expansions, as presented by Mikhailov and Cotta (1996). The results will be compared with known solutions in order to validate the proposed methodology. Table 1 presents reservoir properties that are common for all the applications presented in this work.

\subsection{One-Dimensional Solution in the Cartesian Coordinate System in an Heterogeneous Reservoir}

For this problem, the HDE and its boundary and initial conditions are written as:

$$
\begin{gathered}
\phi c_{\mathrm{t}} \frac{\partial p^{*}(x, t)}{\partial t}=\frac{\partial}{\partial x}\left[\frac{k(x)}{\mu} \frac{\partial p^{*}(x, t)}{\partial x}\right]+\frac{q}{L_{y} L_{z}} \delta\left(x-x_{\mathrm{p}}\right), 0<x<L_{x}, t>0 \\
\frac{\partial p^{*}(x, t)}{\partial x}=0, \quad x=0, t>0 \\
p^{*}(x, t)=0, \quad x=L_{x}, t>0 \\
p^{*}(x, t)=0, \quad 0<x<L_{x}, t=0
\end{gathered}
$$

where $p^{*}(x, t)=p(x, t)-p_{\mathrm{i}}$ is a filter that homogenizes the boundary conditions, improving the convergence of the eigenfunction expansion.

Table 1 Dataset for the applications presented in this work

\begin{tabular}{lll}
\hline Parameter & SI units & Field units \\
\hline Porosity, $\phi$ & $20 \%$ & $20 \%$ \\
Total compressibility, $c_{\mathrm{t}}$ & $1 \times 10^{-9} \mathrm{~Pa}^{-1}$ & $1 \times 10^{-4} \mathrm{bar}^{-1}$ \\
Viscosity, $\mu$ & $10^{-3} \mathrm{~Pa} \mathrm{~s}$ & $1 \mathrm{cP}$ \\
Initial pressure, $p_{i}$ & $5 \times 10^{7} \mathrm{~Pa}$ & $500 \mathrm{bar}$ \\
\hline
\end{tabular}




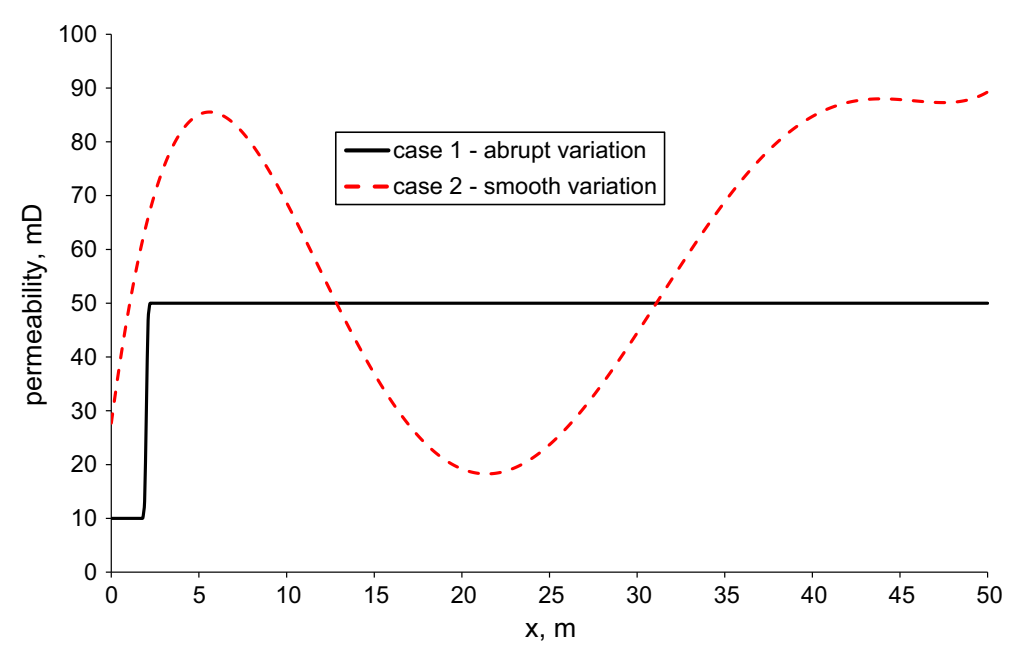

Fig. 1 Reservoir permeabilities considered in the Cartesian coordinate system applications

The permeability $k(x)$ can be represented by any continuous differentiable function. Figure 1 presents the two different cases of reservoir permeabilities that will be considered: (1) abrupt reduction of reservoir permeability near the wellbore, aiming to represent formation damage in the near-wellbore region; (2) smooth permeability variation represented by a fifthorder polynomial.

For the first case, $k(x)$ is represented by the following function:

$$
k(x)=k_{1}+\left(k_{2}-k_{1}\right)\left(\frac{1}{2}+\frac{1}{2} \operatorname{erf}\left(\omega \cdot\left(x-x_{c}\right)\right)\right)
$$

with $k_{1}=10 \mathrm{mD}, k_{2}=50 \mathrm{mD}, \omega=10 \mathrm{~m}^{-1}$ and $x_{c}=2 \mathrm{~m}$. Equation (14) is a continuous approximation of the Heaviside step function. For the second case, $k(x)$ is given by:

$$
\begin{aligned}
k(x)= & 27.6956+24.4593 x-3.28835 x^{2}+0.152226 x^{3}-0.00289167 x^{4} \\
& +1.95333 \times 10^{-6} x^{5}
\end{aligned}
$$

Considering the parameters presented in Table 1 and $x_{\mathrm{p}}=0 \mathrm{~m}, L_{z}=2 \mathrm{~m}, L_{y}=500$ and $L_{x}=50 \mathrm{~m}$, the transient pressure response for Cases 1 and 2 can be obtained by following the procedure presented in Sect. 3 (see "Appendix 1" for the eigenvalues, eigenfunctions and norms of the auxiliary eigenvalue problem).

Case 1: Abrupt permeability variation.

Figure 2 presents the transient pressure behavior at the wellbore position $\left(x=x_{\mathrm{p}}\right)$ for different numbers of terms in the eigenfunction expansion.

As can be seen in Fig. 2, to the graph scale, the solution is already converged with 100 terms in the eigenfunction expansion. In order to check the validity of the results obtained by the approach presented in this work, Fig. 3 shows the pressure distribution throughout the reservoir calculated by: (1) GITT with 300 terms in the eigenfunction expansion and (2) commercial finite difference numerical scheme (CMG's IMEX ${ }^{\mathrm{TM}}$ ).

As can be seen in Fig. 3, the transient pressure response calculated by the GITT is in excellent agreement with the results calculated by the finite difference numerical scheme throughout the whole domain, confirming the accuracy of the results obtained by the GITT. 


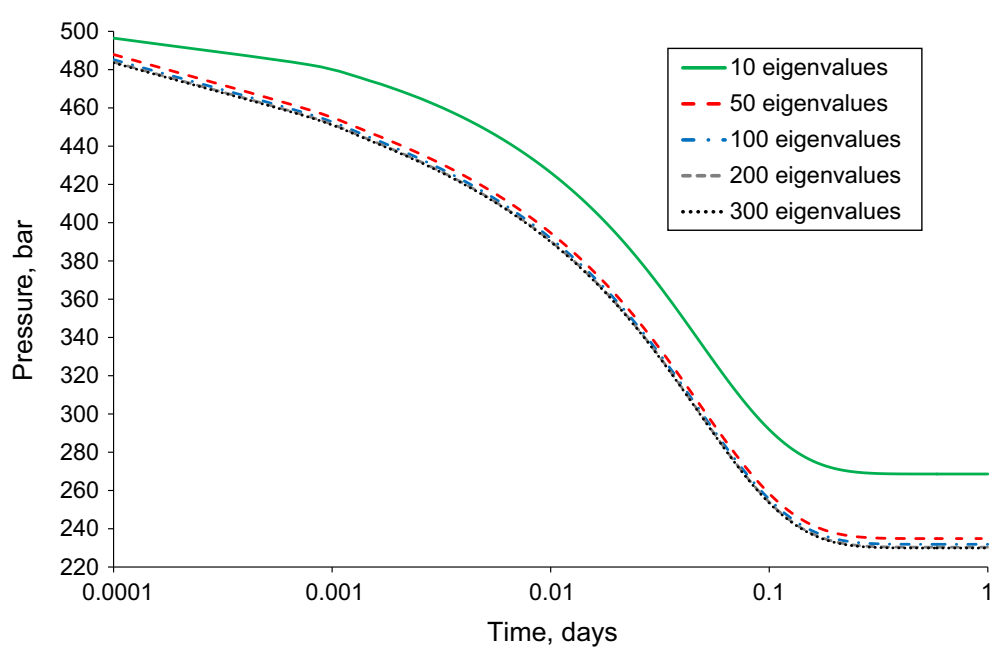

Fig. 2 Transient pressure behavior at $x=x_{\mathrm{p}}$ with different truncation orders

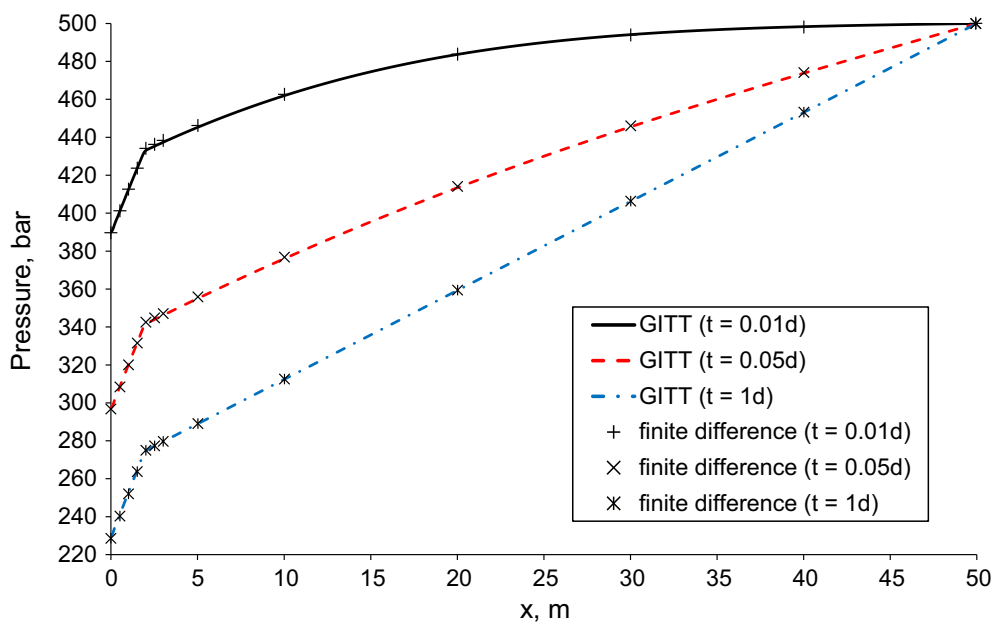

Fig. 3 Pressure throughout the reservoir (Case 1)

The convergence behavior of the GITT solution for time $t=0.01$ days in different positions throughout the reservoir is presented in Table 2.

The results presented in Table 2 show a good convergence behavior of the infinite series, even close to $x=2 \mathrm{~m}$, where the steep permeability variations take place in the reservoir. It can also be observed that the convergence progress is slower close to the source term $\left(x=x_{\mathrm{p}}=0 \mathrm{~m}\right)$, as already mentioned by Hovanessian (1961).

Pressure derivative analysis was introduced by Bourdet and Ayoub (1989) and is nowadays widely used for the interpretation of pressure transient tests and reservoir characterization. The solution proposed in this work can be used to obtain exact solutions for the derivative of pressure with time by applying the operator $\partial / \partial t$ to Eq. 6 . Table 3 presents an analysis of the convergence of the pressure derivative calculated by the GITT at the wellbore position $\left(x=x_{\mathrm{p}}\right)$. 
Table 2 Convergence behavior of pressure at $t=0.01$ days (Case 1)

Table 3 Convergence behavior of pressure derivative at $x=x_{\mathrm{p}}$ (Case 1)

\begin{tabular}{|c|c|c|c|c|c|}
\hline \multirow[t]{3}{*}{$x(\mathrm{~m})$} & \multicolumn{5}{|c|}{ Pressure (bar) } \\
\hline & \multicolumn{5}{|c|}{ Number of terms $(N)$} \\
\hline & 10 & 50 & 100 & 200 & 300 \\
\hline 0 & 426.19 & 394.67 & 391.83 & 390.39 & 389.95 \\
\hline 2 & 429.76 & 432.45 & 432.76 & 432.94 & 432.97 \\
\hline 5 & 443.47 & 445.07 & 445.22 & 445.23 & 445.23 \\
\hline 10 & 461.07 & 461.84 & 461.89 & 461.92 & 461.92 \\
\hline 20 & 483.02 & 483.62 & 483.65 & 483.67 & 483.67 \\
\hline 30 & 493.77 & 494.10 & 494.11 & 494.12 & 494.12 \\
\hline 40 & 498.19 & 498.31 & 498.32 & 498.32 & 498.32 \\
\hline 50 & 500.00 & 500.00 & 500.00 & 500.00 & 500.00 \\
\hline
\end{tabular}

\begin{tabular}{|c|c|c|c|c|c|}
\hline \multirow[t]{3}{*}{$t$ (days) } & \multicolumn{5}{|c|}{$\partial p / \partial t \times 10^{3}(\mathrm{bar} / \mathrm{s})$} \\
\hline & \multicolumn{5}{|c|}{ Number of terms $(N)$} \\
\hline & 10 & 50 & 100 & 200 & 300 \\
\hline 0.001 & -145.99 & -213.24 & -216.89 & -218.48 & -218.71 \\
\hline 0.01 & -44.91 & -46.26 & -46.36 & -46.40 & -46.40 \\
\hline 0.02 & -31.51 & -32.00 & -32.03 & -32.05 & -32.05 \\
\hline 0.1 & -5.66 & -5.73 & -5.73 & -5.73 & -5.73 \\
\hline 0.2 & -0.69 & -0.70 & -0.70 & -0.70 & -0.70 \\
\hline 1 & 0.00 & 0.00 & 0.00 & 0.00 & 0.00 \\
\hline
\end{tabular}

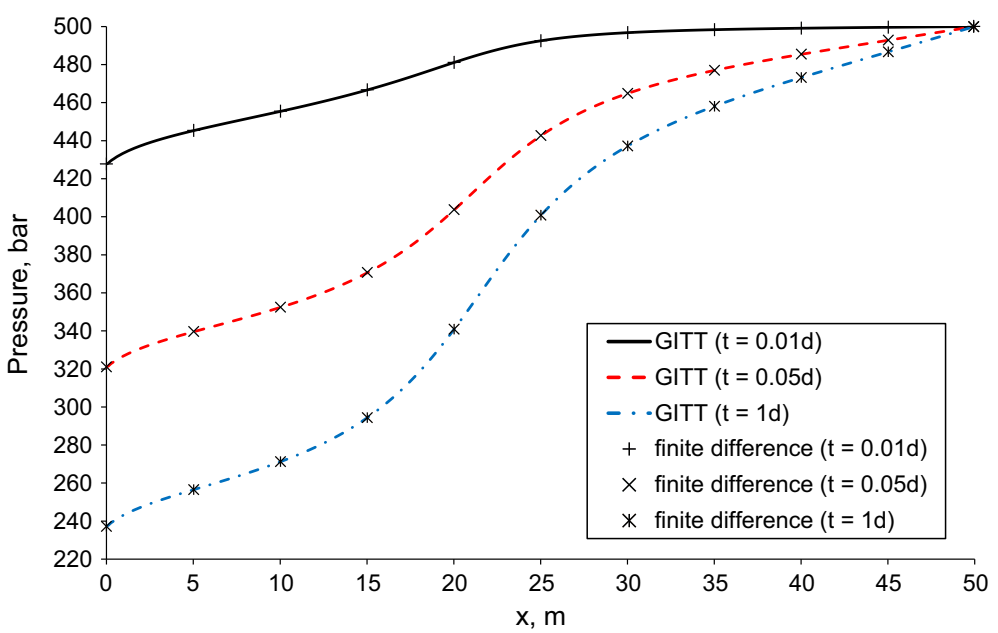

Fig. 4 Pressure throughout the reservoir (Case 2)

The analysis of Table 3 shows that $\partial p / \partial t$ has an excellent convergence behavior and that convergence of $\partial p / \partial t$ can be obtained even at the wellbore position $\left(x=x_{\mathrm{p}}\right)$. 
Table 4 Convergence behavior of pressure at $t=0.01$ day (Case 2)

Table 5 Convergence behavior of pressure derivative at $x=x_{\mathrm{p}}$ (Case 2)

\begin{tabular}{|c|c|c|c|c|c|c|}
\hline \multirow[t]{3}{*}{$x(\mathrm{~m})$} & \multicolumn{6}{|c|}{ Pressure (bar) } \\
\hline & \multicolumn{6}{|c|}{ Number of terms $(N)$} \\
\hline & 10 & 50 & 100 & 200 & 300 & 400 \\
\hline 0 & 434.59 & 429.37 & 428.42 & 427.94 & 427.78 & 427.70 \\
\hline 5 & 444.86 & 445.24 & 445.25 & 445.25 & 445.25 & 445.25 \\
\hline 10 & 455.27 & 455.37 & 455.37 & 455.38 & 455.38 & 455.38 \\
\hline 15 & 466.77 & 466.62 & 466.61 & 466.61 & 466.61 & 466.61 \\
\hline 20 & 480.97 & 481.05 & 481.05 & 481.05 & 481.05 & 481.05 \\
\hline 25 & 492.14 & 492.49 & 492.50 & 492.50 & 492.50 & 492.50 \\
\hline 30 & 496.90 & 496.79 & 496.78 & 496.78 & 496.78 & 496.78 \\
\hline 35 & 498.24 & 498.35 & 498.35 & 498.35 & 498.35 & 498.35 \\
\hline 40 & 499.14 & 499.12 & 499.12 & 499.12 & 499.12 & 499.12 \\
\hline 45 & 499.58 & 499.60 & 499.60 & 499.60 & 499.60 & 499.60 \\
\hline 50 & 500.00 & 500.00 & 500.00 & 500.00 & 500.00 & 500.00 \\
\hline
\end{tabular}

\begin{tabular}{|c|c|c|c|c|c|}
\hline \multirow[t]{3}{*}{$t$ (days) } & \multicolumn{5}{|c|}{$\partial p / \partial t \times 10^{3}(\mathrm{bar} / \mathrm{s})$} \\
\hline & \multicolumn{5}{|c|}{ Number of terms $(N)$} \\
\hline & 10 & 50 & 100 & 200 & 300 \\
\hline 0.001 & -113.42 & -116.03 & -116.12 & -116.13 & -116.14 \\
\hline 0.01 & -47.78 & -47.96 & -47.96 & -47.96 & -47.96 \\
\hline 0.02 & -36.71 & -36.81 & -36.81 & -36.81 & -36.81 \\
\hline 0.1 & -7.10 & -7.13 & -7.13 & -7.13 & -7.13 \\
\hline 0.2 & -0.93 & -0.93 & -0.93 & -0.93 & -0.93 \\
\hline 1 & 0.00 & 0.00 & 0.00 & 0.00 & 0.00 \\
\hline
\end{tabular}

Case 2: Smooth permeability variation.

Figure 4 shows the pressure distribution throughout the reservoir calculated by: (1) GITT with 300 terms in the eigenfunction expansion and (2) commercial finite difference numerical scheme.

As can be seen in Fig. 4, the transient pressure response calculated by the GITT is in excellent agreement with the results calculated by the finite difference numerical scheme, confirming the accuracy of the results obtained by the GITT. The convergence behavior of the GITT solution for time $t=0.01$ days in different positions throughout the reservoir is presented in Table 4.

An analysis of the convergence of $\partial p / \partial t$ at the wellbore position $\left(x=x_{\mathrm{p}}\right)$ is shown in Table 5, where it can be observed that $\partial p / \partial t$ has an excellent convergence behavior, even at the well's position $\left(x=x_{\mathrm{p}}\right)$.

In addition to $\partial p / \partial t$, the calculation of $\partial p / \partial x$ can be useful for practical applications, for example, the calculation of flow velocities throughout the reservoir through Darcy's law. The GITT can be used to attain converged solutions of $\partial p / \partial x$ as presented in "Appendix 3."

To illustrate the capabilities of the GITT to solve the HDE, Fig. 5 presents the pressure distribution for the permeability considered in Case 2 (GITT with 300 terms in the eigen- 


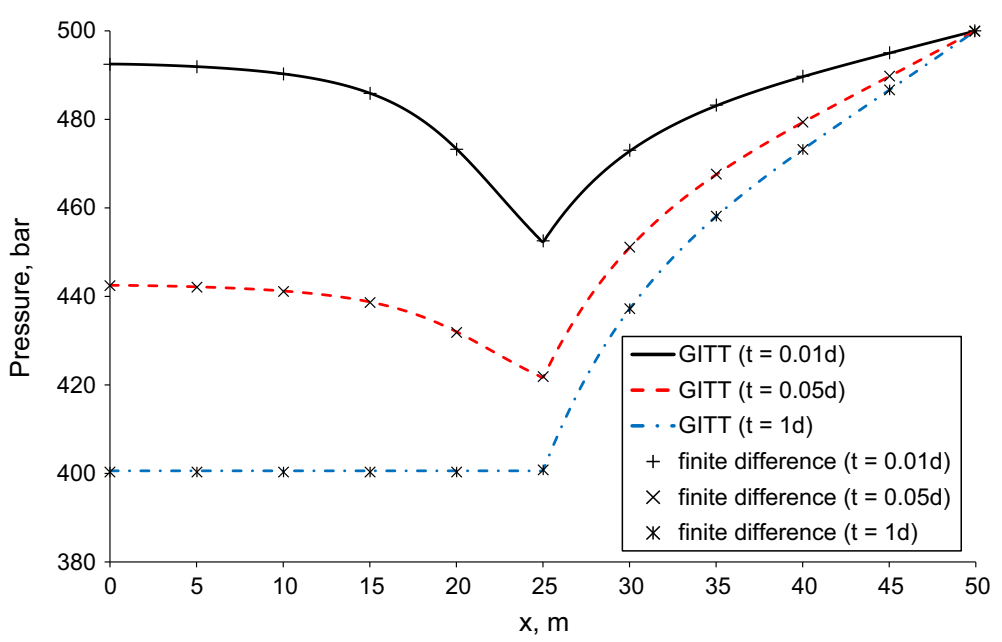

Fig. 5 Pressure throughout the reservoir (Case 2, $x_{\mathrm{p}}=25 \mathrm{~m}$ )

function expansion), but instead of placing the well at the system boundary $\left(x_{\mathrm{p}}=0\right)$, the well now is placed at $x_{\mathrm{p}}=25 \mathrm{~m}$.

As can be seen in Fig. 5, the proposed solution of the HDE easily handles different positions of the source term throughout the reservoir, being it possible to use the same solution procedure to consider several wellbores situated in arbitrary positions throughout the reservoir, as presented by Couto et al. (2011).

\subsection{One-Dimensional Solution in the Radial Coordinate System in an Heterogeneous Reservoir}

For this problem, the HDE and its boundary and initial conditions are written as:

$$
\begin{gathered}
\phi c_{\mathrm{t}} \frac{\partial p^{*}(r, t)}{\partial t}=\frac{1}{r} \frac{\partial}{\partial r}\left[r \frac{k(r)}{\mu} \frac{\partial p^{*}(r, t)}{\partial r}\right]+\frac{q}{2 \pi L_{z}} \delta\left(r-r_{\mathrm{p}}\right), 0<r<r_{e}, t>0 \\
\frac{\partial p^{*}(r, t)}{\partial r}=0, \quad r=0, t>0 \\
\frac{\partial p^{*}(r, t)}{\partial r}=0, \quad r=r_{e}, t>0 \\
p^{*}(r, t)=0, \quad 0<r<r_{e}, t=0
\end{gathered}
$$

where $p^{*}(r, t)=p(r, t)-p_{\mathrm{i}}$ is a filter that homogenizes the boundary conditions, improving the convergence of the eigenfunction expansion.

The permeability $k(r)$ can be represented by any continuous differentiable function. An application considering a smooth permeability variation throughout the reservoir (Fig. 6) represented by a fifth-order polynomial is going to be considered:

$$
\begin{aligned}
k(r)= & 67.2419-0.562439 r+9.25807 \times 10^{-3} r^{2}-6.92901 \times 10^{-5} r^{3} \\
& +2.00515 \times 10^{-7} r^{4}-1.89480 \times 10^{-10} r^{5}
\end{aligned}
$$

Considering the parameters already presented in Table 1 and $L_{z}=100 \mathrm{~m}, r_{e}=500 \mathrm{~m}$, the transient pressure response can be obtained by following the procedure presented in Sect. 3 


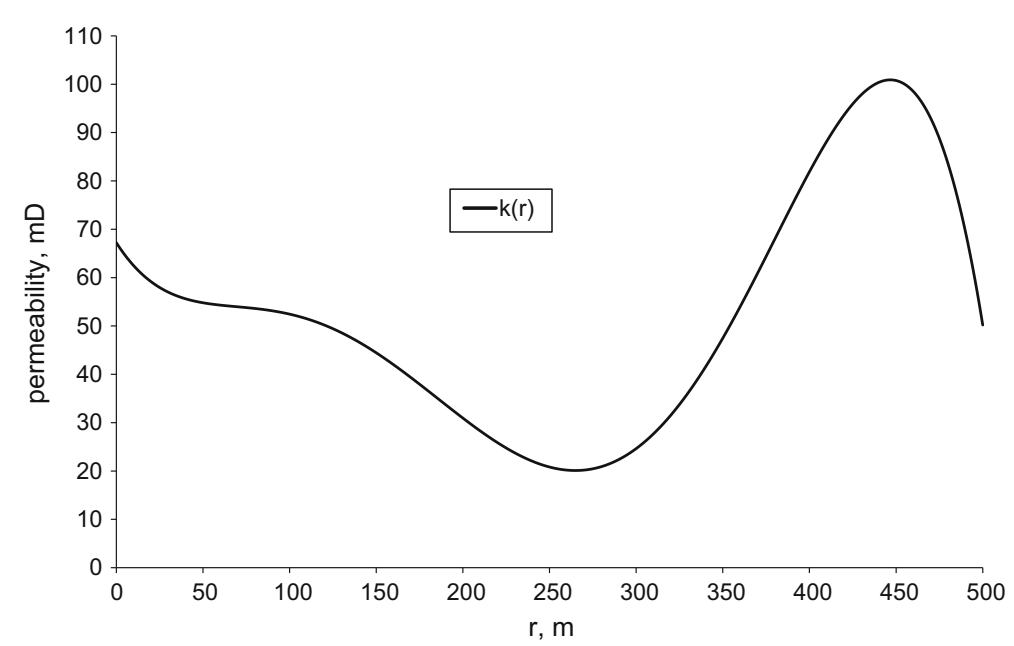

Fig. 6 Reservoir permeability considered in the radial coordinate system application

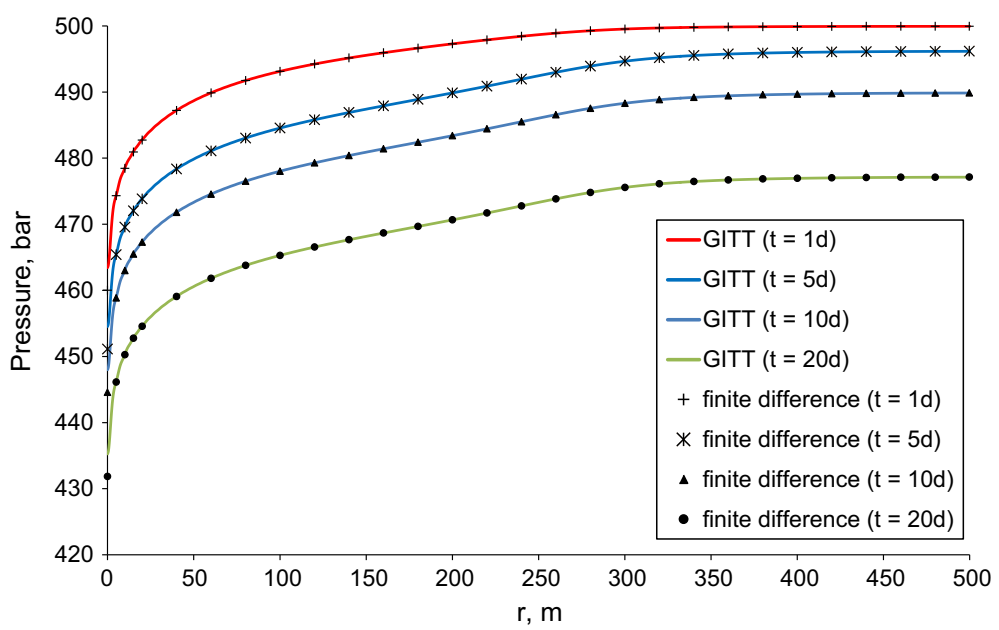

Fig. 7 Pressure throughout the reservoir in a radial coordinate system

(see "Appendix 2" for the eigenvalues, eigenfunctions and norms of the auxiliary eigenvalue problem).

Figure 7 shows the pressure distribution throughout the reservoir calculated by: (1) GITT with 250 terms in the eigenfunction expansion and (2) commercial finite difference numerical scheme.

As can be seen in Fig. 7, the transient pressure response calculated by the GITT is in excellent agreement with the results calculated by the finite difference numerical scheme, confirming the accuracy of the results obtained by the GITT and its ability to properly represent the reservoir heterogeneities. The convergence behavior of the GITT solution for time $t=10$ days in different positions throughout the reservoir is presented in Table 6 .

The results presented in Table 6 show that the solution has a good convergence behavior for positions far from the source term (well's position). For positions close to the source term, 
Table 6 Convergence behavior of pressure at $t=10$ days

\begin{tabular}{|c|c|c|c|c|c|c|}
\hline \multirow[t]{3}{*}{$r(\mathrm{~m})$} & \multicolumn{6}{|c|}{ Pressure (bar) } \\
\hline & \multicolumn{6}{|c|}{ Number of terms $(N)$} \\
\hline & 10 & 50 & 100 & 150 & 200 & 250 \\
\hline 0.1 & 466.50 & 456.98 & 453.09 & 450.83 & 449.24 & 448.00 \\
\hline 1 & 466.50 & 457.05 & 453.37 & 451.44 & 450.29 & 449.60 \\
\hline 5 & 466.58 & 458.68 & 458.38 & 459.22 & 459.24 & 458.90 \\
\hline 10 & 466.82 & 462.43 & 463.31 & 462.96 & 463.16 & 463.02 \\
\hline 50 & 472.54 & 473.26 & 473.34 & 473.31 & 473.33 & 473.32 \\
\hline 100 & 478.28 & 478.04 & 478.03 & 478.03 & 478.02 & 478.02 \\
\hline 200 & 483.51 & 483.40 & 483.40 & 483.39 & 483.39 & 483.39 \\
\hline 300 & 488.42 & 488.31 & 488.30 & 488.30 & 488.30 & 488.30 \\
\hline 400 & 489.72 & 489.68 & 489.68 & 489.68 & 489.68 & 489.68 \\
\hline 500 & 489.90 & 489.85 & 489.85 & 489.85 & 489.85 & 489.85 \\
\hline
\end{tabular}

Table 7 Convergence behavior of pressure derivative at $r=0.1 \mathrm{~m}$

\begin{tabular}{|c|c|c|c|c|c|c|}
\hline \multirow[t]{3}{*}{$t$ (days) } & \multicolumn{6}{|c|}{$\partial p / \partial t \times 10^{3}(\mathrm{bar} / \mathrm{s})$} \\
\hline & \multicolumn{6}{|c|}{ Number of terms $(N)$} \\
\hline & 10 & 50 & 100 & 150 & 200 & 250 \\
\hline 0.001 & -3.3076 & -32.1662 & -33.5731 & -33.5248 & -33.5169 & -33.5143 \\
\hline 0.01 & -2.3167 & -3.5809 & -3.5777 & -3.5774 & -3.5773 & -3.5773 \\
\hline 0.02 & -1.6430 & -1.8377 & -1.8370 & -1.8369 & -1.8369 & -1.8369 \\
\hline 0.1 & -0.3947 & -0.3895 & -0.3894 & -0.3894 & -0.3894 & -0.3894 \\
\hline 0.2 & -0.2020 & -0.2009 & -0.2009 & -0.2009 & -0.2009 & -0.2009 \\
\hline 1 & -0.0531 & -0.0528 & -0.0528 & -0.0528 & -0.0528 & -0.0528 \\
\hline 10 & -0.0148 & -0.0148 & -0.0148 & -0.0148 & -0.0148 & -0.0148 \\
\hline 15 & -0.0147 & -0.0147 & -0.0147 & -0.0147 & -0.0147 & -0.0147 \\
\hline 20 & -0.0147 & -0.0147 & -0.0147 & -0.0147 & -0.0147 & -0.0147 \\
\hline
\end{tabular}

convergence progress becomes slower and is not attained, at least for 250 eigenvalues in the expansion.

In spite of pressure convergence not being satisfactory at the well's positions, the convergence of the pressure derivative with time is much faster, as can be observed by the analysis of $\partial p / \partial t$ convergence behavior at the position $x=0.1 \mathrm{~m}$ for different times, presented in Table 7.

The analysis of Table 7 shows that $\partial p / \partial t$ has an excellent convergence behavior at the well's position, even for very short times (few minutes). Figure 8 shows the plot of $t \cdot \partial P / \partial t$ versus $t$ in a log-log scale (a plot commonly used in well test analysis for reservoir characterization) at the well's position ( $x=0.1 \mathrm{~m}$ ) as calculated by: (1) GITT with different numbers of terms in the eigenfunction expansion and (2) commercial finite difference numerical scheme.

The analysis of Fig. 8 shows that the convergence of $t \times \partial P / \partial t$ depends on time. For $t=0.01$ days, the solution is converged, at least to the graph scale, with only 50 terms in the eigenfunction expansion, and for short times as $t=0.0001 \mathrm{~d}, 200$ terms are necessary 


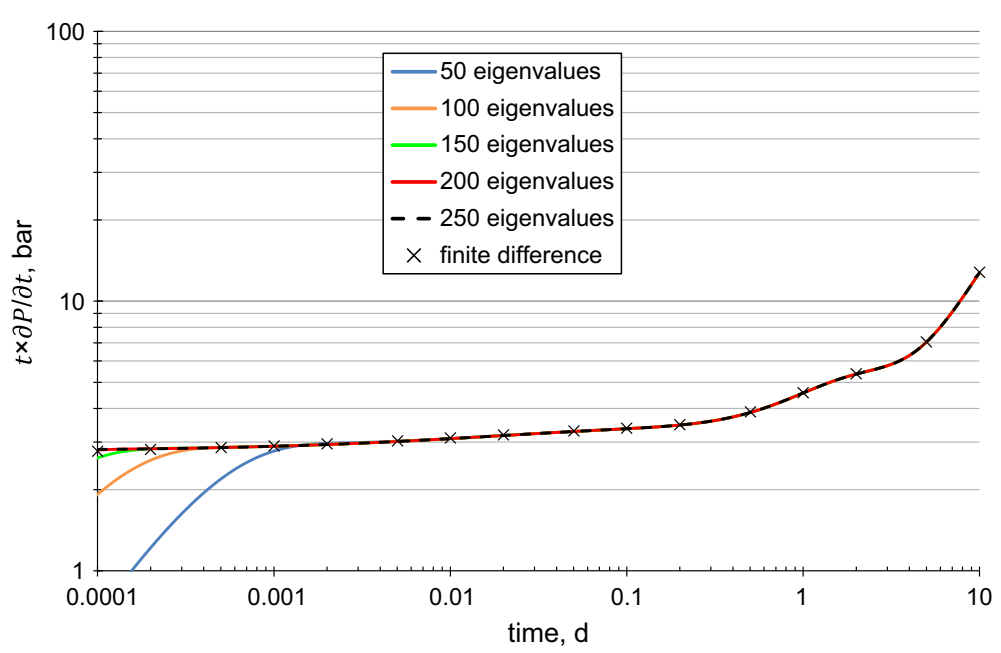

Fig. 8 Log-log derivative plot for the problem of Sect. 4.2

to attain convergence of the solution. It is also possible to observe that the results perfectly match the ones obtained by the finite difference numerical scheme.

\section{Conclusions}

A comprehensive and rigorous solution of the transient hydraulic diffusivity equation in heterogeneous porous media using the GITT is presented. The presented general solution and its derivation are valid for multi-dimensional problems in any orthogonal coordinate system, with generalized boundary and initial conditions. The solution presented is valid for all flow regimes and allows the calculation of pressure throughout the reservoir and not only for specific positions inside the porous media.

Applications of the proposed solution to one-dimensional problems in the Cartesian coordinate system are presented considering two different cases of reservoir permeability: (1) abrupt permeability variation represented by a continuous approximation of the Heaviside step function and (2) smooth permeability variation represented by a fifth-order polynomial. The generality of the proposed solution is explored by applying the same solution method to a one-dimensional problem in the radial coordinate system with heterogeneous permeability represented by a fifth-order polynomial. For all the examples presented, the accuracy of the GITT results is checked with results obtained with a finite difference numerical scheme, showing that the GITT solution is able to properly represent the reservoir heterogeneities considered. Convergence analysis of the pressure was presented, showing that the solution is stable with controlled accuracy, being the convergence slower for positions near the well. The GITT allows the obtainment of time derivatives of pressure analytically, without the use of any numerical procedure. The convergence behavior of the time derivatives is presented, showing that the analytical representation of the time derivatives has an excellent convergence behavior, even at the well's position. For the radial coordinate system application, the loglog derivative plot is presented, illustrating an example of the application of the GITT to the interpretation of transient well test data. Although not explored in this work, the application of the proposed general solution can be extended to two- and three-dimensional problems. 
The GITT allows the establishment of benchmark results for transient flow problems in heterogeneous porous media and presents itself as a new tool for the analysis of buildup, drawdown and interference test data associated with pressure transient analysis in heterogeneous reservoirs.

Acknowledgments The first author thanks Petrobras for support in the development of this work. The authors sincerely thank Prof. Carolina Naveira-Cotta for sharing her knowledge and expertise with integral transforms during the development of this work. The second and third authors also acknowledge $\mathrm{CNP}_{\mathrm{q}}$ and FAPERJ for the continuous support of their research projects during the past years.

\section{Appendix 1}

The eigenvalues, eigenfunctions and norms of the auxiliary eigenvalue problem used to solve the problem given by Eqs. (13a) through (13d) are:

$$
\begin{aligned}
\Omega_{n(x)} & =\cos \left(\lambda_{n} x\right) \\
\lambda_{n} & =\frac{2 \pi(n-1)}{2 L_{x}}, \quad n=1,2,3 \ldots \\
N_{\Omega_{n}} & =L_{x} / 2
\end{aligned}
$$

\section{Appendix 2}

The eigenvalues, eigenfunctions and norms of the auxiliary eigenvalue problem used to solve the problem given by Eqs. 16a-16d are:

$$
\begin{aligned}
\Omega_{n(r)} & =J_{0}\left(\lambda_{n} r\right) \\
\lambda_{n} & =\text { non negative roots of }\left(J_{0}\left(\lambda_{n} r_{e}\right)=0\right) \\
N_{\Omega_{n}} & =\frac{r_{e}^{2} J_{0}^{2}\left(\lambda_{n} r_{e}\right)}{2}
\end{aligned}
$$

\section{Appendix 3: Integral Balance}

The integral balance can be used as a tool to accelerate the convergence of the eigenfunction expansions, as presented by Scofano Neto et al. (1990) and Leiroz and Cotta (1990). This technique is used in order to attain a faster convergence of the spatial derivatives of pressure. The integral balance is based on the integration of the original partial differential equation (Eq. 4a) in the spatial domain followed by a manipulation of the boundary conditions. Applying the operator $\int_{0}^{x} \mathrm{~d} x$ in Eq. $4 \mathrm{a}$ :

$$
\begin{aligned}
\frac{\partial}{\partial t}\left[\int_{0}^{x} \phi(x) c_{\mathrm{t}} p(x, t) \mathrm{d} x\right]= & \left.\frac{k(x)}{\mu} \frac{\partial p(x, t)}{\partial x}\right|_{x=x}-\left.\frac{k(x)}{\mu} \frac{\partial p(x, t)}{\partial x}\right|_{x=0} \\
& +\int_{0}^{x} W_{\mathrm{p}}(x, t) \mathrm{d} x
\end{aligned}
$$

substituting a boundary condition with known value of $\partial p(x, t) / \partial x$ in Eq. 24, one can obtain an expression for $\partial p(x, t) / \partial x$ with excellent convergence behavior. 

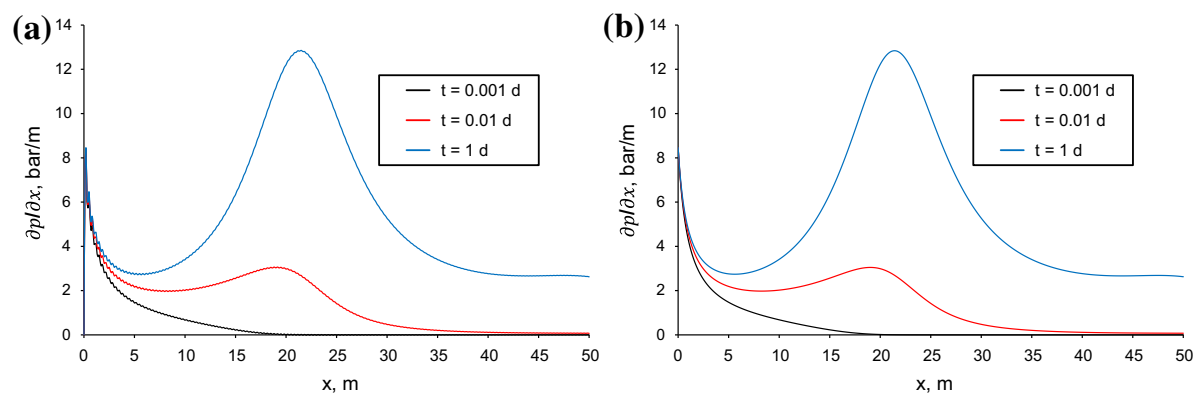

Fig. 9 Pressure derivative throughout the reservoir calculated by: a deriving Eq. 6 and $\mathbf{b}$ integral balance

Table 8 Convergence behavior of spatial pressure derivative at $t=0.01$ days (Case 2) calculated using the spatial derivative of Eq. 6

Table 9 Convergence behavior of spatial pressure derivative at $t=0.01$ days (Case 2) calculated using the integral balance

\begin{tabular}{|c|c|c|c|c|c|}
\hline \multirow[t]{3}{*}{$x(\mathrm{~m})$} & \multicolumn{5}{|c|}{$\partial p / \partial x(\mathrm{bar} / \mathrm{m})$} \\
\hline & \multicolumn{5}{|c|}{ Number of terms $(N)$} \\
\hline & 5 & 10 & 25 & 50 & 100 \\
\hline 0.1 & 0.04132 & 0.11324 & 0.41233 & 1.05652 & 2.50599 \\
\hline 1 & 0.41149 & 1.10256 & 3.50305 & 5.70715 & 3.96026 \\
\hline 5 & 1.85822 & 2.86902 & 2.16121 & 2.39140 & 2.08440 \\
\hline 10 & 2.73776 & 1.62444 & 2.22406 & 1.91202 & 1.96594 \\
\hline 20 & 2.08025 & 2.53958 & 2.78671 & 2.87803 & 2.93297 \\
\hline 30 & 0.88337 & 0.28922 & 0.56681 & 0.42765 & 0.45157 \\
\hline 40 & -0.04235 & -0.00211 & 0.05662 & 0.08316 & 0.09908 \\
\hline 50 & 0.24070 & -0.03463 & 0.13227 & 0.04428 & 0.05923 \\
\hline
\end{tabular}

\begin{tabular}{|c|c|c|c|c|c|}
\hline \multirow[t]{3}{*}{$x(\mathrm{~m})$} & \multicolumn{5}{|c|}{$\partial P / \partial x(\mathrm{bar} / \mathrm{m})$} \\
\hline & \multicolumn{5}{|c|}{ Number of terms $(N)$} \\
\hline & 5 & 10 & 25 & 50 & 100 \\
\hline 0.1 & 7.75867 & 7.75788 & 7.75777 & 7.75776 & 7.75776 \\
\hline 1 & 4.59249 & 4.58763 & 4.58699 & 4.58695 & 4.58695 \\
\hline 5 & 2.20154 & 2.19003 & 2.18897 & 2.18889 & 2.18888 \\
\hline 10 & 2.04284 & 2.02551 & 2.02291 & 2.02274 & 2.02271 \\
\hline 20 & 3.20047 & 3.00958 & 2.99239 & 2.99144 & 2.99125 \\
\hline 30 & 0.51889 & 0.48201 & 0.47740 & 0.47707 & 0.47701 \\
\hline 40 & 0.13231 & 0.11760 & 0.11611 & 0.11599 & 0.11596 \\
\hline 50 & 0.08391 & 0.07627 & 0.07517 & 0.07507 & 0.07505 \\
\hline
\end{tabular}

Figure 9 presents $\partial p(x, t) / \partial x$ for the problem presented in Case 2 as calculated by two ways: (a) deriving the solution given by Eq. 6 and using 300 terms in the eigenfunction expansion and (b) by applying the integral balance with 100 terms in the eigenfunction expansion. 
The convergence behavior of $\partial p / \partial x$ as calculated by deriving Eq. 6 and by using the integral balance is presented in Tables 8 and 9, respectively.

As can be seen in the analysis of Fig. 9 and Tables 8 and 9, the integral balance greatly enhances the convergence behavior of $\partial p / \partial x$ and can be used to attain converged solutions of the flow velocities during transient flow in heterogeneous porous media.

\section{References}

Almeida, A., Cotta, R.: Integral transform methodology for convection-diffusion problems in petroleum reservoir engineering. Int. J. Heat Mass Transf. 38, 3359-3367 (1995)

Bourdet, D., Ayoub, J., Pirard, Y.: Use of pressure derivative in well test interpretation. SPE J. 4, $293-302$ (1989)

Carslaw, H., Jaeger, J.: Conduction of Heat in Solids. Oxford Science Publications (1959)

Cotta, R.: Integral Transforms in Computational Heat and Fluid Flow. CRC Press, Boca Raton (1993)

Cotta, R.: Benchmark results in computational heat and fluid flow: the integral transform method. Int. J. Heat Mass Transf. 37, 381-393 (1994)

Couto, P., Moreira, R., Marsilli, M.: A General Analytical Solution for the Multidimensional Transient Linear Hydraulic Diffusivity Equation in Heterogeneous and Anisotropic Porous Media (2011)

Hovanessian, S.A.: Pressure studies in bounded reservoirs. SPE J. 1, 223-228 (1961)

Leiroz, A.J.K., Cotta, R.M.: Convergence Enhancement of Eigenfuction Expansions for Nonhomogeneous Elliptic Diffusion Problems. III Encontro Nacional de Ciências Térmicas, ENCIT 90, vol. 1, pp. 335-340, Itapema, SC, December (1990)

Matthews, C., Russell, D.: Pressure Buildup and Flow Tests in Wells. Storm Printing Corp, Dallas (1967)

Mikhailov, M., Cotta, R.: Ordering rules for double and triple eigenseries in the solution of multidimensional heat and fluid flow problems. Int. Commun. Heat Mass Transf. 23, 299-303 (1996)

Mikhailov, M., Ozisik, M.: Unified Analysis and Solutions of Heat and Mass Diffusion. Wiley, New York (1984)

Naveira-Cotta, C., Cotta, R., Orlande, H., Fudym, O.: Eigenfunction expansions for transient diffusion in heterogeneous media. Int. J. Heat Mass Transf. 52, 5029-5039 (2009)

Ozisik, M.: Heat Conduction. Wiley, New York (1993)

Rahman, N., Bentsen, R.: Use of an Integral Transform Technique for Comprehensive Solutions to Transient Flow Problems in Homogeneous Domains. PETSOC paper presented at the Canadian International Petroleum Conference, Calgary, Alberta, 4-8 June. http://dx.doi.org/10.2118/2000-042 (2000)

Rahman, N., Bentsen, R.: Comprehensive Solutions for Transient-Flow Problems in 3D Homogeneous Domains. SPE paper presented at the SPE Middle East Oil Show, Manama, Bahrain, 17-20 March. http://dx.doi.org/10.2118/68139-MS (2001)

Scofano Neto, F., Cotta, R., Mikhailov, M.: Alternative Approach to the Integral Transform Solution of Nonhomogeneous Diffusion Problems. Int. Conf. on Advanced Computational Methods in Heat Transfer, vol. 1, pp. 39-50, Southampton, UK, July (1990)

Wolfram, S.: The Mathematica Book, 5.2nd edn. Wolfram Media, Cambridge (2005) 